\title{
Interpretation of Modified Electromagnetic Theory and Maxwell's Equations on the basis of Charge Variation
}

\author{
Asif Ali Laghari \\ Department of Electronics Engineering, \\ Mehran University of Engineering and Technology, \\ Jamshoro, Sindh, Pakistan
}

\begin{abstract}
Electromagnetic waves are the analytical solutions of Maxwell's equations that represent one of the most elegant and concise ways to state the fundamentals of electricity and magnetism. From them one can develop most of the working relationships in the electric and magnetic fields. Considering deeply the effect of charge variation in Maxwell's equations for time varying electric and magnetic fields of charges in moving inertial frame, the magnitude of charge particles vary according to Asif's equation of charge variation. Consequently the Maxwell's equations give different results to an observer measuring at rest. This research paper explained the effect of charge variation in Classical Electromagnetic theory, Maxwell's equations, Coulumb's law, Lorentz force law when we are referring to any inertial frame
\end{abstract}

\section{General Terms:}

Differential Equations, Lorentz Force

\section{Keywords:}

Maxwell's Equation, Electric Field, Electromagnetic Force, Electromagnetic Waves

\section{INTRODUCTION}

Maxwell's equation are a set of partial differential equations that, together with the Lorentz force law, form the foundation of classical electrodynamics, classical optics, and electric circuits. These fields in turn underlie modern electrical and communications technologies. Maxwell's equations describe how electric and magnetic fields are generated and altered by each other and by charges and currents. They are named after the Scottish physicist and mathematician James Clerk Maxwell who published an early form of those equations between 1861 and 1862 [1]. The "microscopic" set of Maxwell's equations uses total charge and total current without considering charge variation when charges are moving with high speed due to relativistic effect on charge [2].

Consequently they gives different result when charges are in moving inertial frame. In this perspective, we have investigated the creation of electromagnetic wave by moving point charge or by time varying electric and magnetic field in the presence of charges at rest, uniform motion or in accelerated frame. The main and important consequence of Modified electromagnetic theory is that it also predicts the charge distorts space-time curvature as Einstein explained mass distorts space-time curvature in his general theory of relativity [3].

There are main two postulate of modified electromagnetic theory.
1. $\frac{\mathrm{e}}{\mathrm{m}}$ Ratio of any charged body with the mass " $\mathrm{m}$ " and charge " $\mathrm{e}$ " is constant for all observers regardless their state of motion.

2. Maxwell's Electromagnetic wave equations of point charge without considering charge variation are not valid for moving frame with respect to rest observer.

\section{ORIGIN OF COULOMB'S FORCE}

It follows from coulomb's law that electrostatic force between two charges is directly proportional to the product of two charges and inversely proportional to the square of the distance between them given by

$$
\mathrm{F}=\frac{\mathrm{kq}_{1} \mathrm{q}_{2}}{\mathrm{r}^{2}}
$$

Where ' $\mathrm{k}$ ' is the coulomb's constant. $\mathrm{K}=\frac{1}{4 \pi \varepsilon_{0}}$. From Maxwell's equation, substituting the value of $\varepsilon_{0}$ in above equation.

$F=\mu_{0} c^{2} \frac{q_{1} q_{2}}{4 \pi r^{2}}$

Let us assume that $\mathrm{q}_{1}$ is the point charge and $\mathrm{q}_{2}$ is fixed charge. Hence above equation can be written as.

$\mathrm{F}=\frac{\mu_{0}}{4 \pi} \frac{\mathrm{q}_{1}\left(\mathrm{q}_{2} \mathrm{c}^{2}\right)}{\mathrm{r}^{2}}$

The factor $\mathrm{q}_{2} \mathrm{c}^{2}$ appearing in the above equation is actual cause of coulomb's force and is responsible for charge distorts space-time curvature as Einstein proposed that factor $\mathrm{mc}^{2}$ appearing in Newton's universal law of gravitation which was further explained by Einstein in his general theory of relativity causes mass distorts space- time curvature.

\subsection{Modification in Lorentz force law}

It is generally expected from intuition that the electromagnetic force exerted on a charged particle should he invariant as observed in different inertial frames. In terms of electric and magnetic fields the electromagnetic force exerted on a particle of charge $\mathrm{q}$ and velocity $\mathrm{v}$ is given by the famous Lorentz force law as $\mathrm{F}=\mathrm{q}(\mathrm{E}+\mathrm{v} \times \mathrm{B})$. By resorting to the Lorentz transformation of space and time, it is known that the two fields together with the velocity transform in such a way that the Lorentz force is exactly identical to that given by the transformation of the time rate of change of kinematic momentum. That is, the Lorentz force law is Lorentz invariant. Furthermore, the wave equations of potential, the continuity equation, and the Lorentz gauge, which are fundamental equations in electromagnetic, can be shown to be Lorentz invariant. Then, Maxwell's equations can be shown to be invariant under the Lorentz transformation [4].

Consequently Lorentz force equation doesn't involve charge variation when the charge particle moves in uniform electric 
and magnetic field and it is Lorentz invariant [5]. According to modern strategy of relativistic charge, Lorentz force equation must involve relativistic charge if charges are in moving frame.

Let us assume that two same charges are moving with the velocity $\mathrm{V}$ along $\mathrm{y}$-axis in uniform electric and magnetic field along $y$-axis and $z$-axis respectively as given in fig. 1. Lorentz force can be calculated by using following equation.

$\mathrm{F}=\mathrm{q}_{0}(\mathrm{E}+\mathrm{V} \times \mathrm{B})$

Where ' $\mathrm{q}_{0}$ ' is rest charge. If the velocity of charge is very high as compared to speed of light for rest observer, So considering the effect of charge variation, replacing ' $\mathrm{q}_{0}$ ' by the relativistic charge ' $q$ '.

Therefore modified equation of Lorentz force is.

$\mathrm{F}=\mathrm{q}(\mathrm{E}+\mathrm{V} \times \mathrm{B})$

\subsection{Lorentz force on continuous charge distribution}

For the continuous charge distribution, suppose that charges are distributed over small volume in moving with constant velocity in electromagnetic field, hence a very small force will act on a very small amount of charge with respect to rest observer, by taking differential.

$$
\mathrm{df}=\mathrm{dq}\left[\gamma(\mathrm{v} \times \mathrm{B})+\mathrm{E}_{0}\right]
$$

Where ' $\gamma^{\prime}$ is Lorentz factor described in Lorentz transformation.

$$
\gamma=\sqrt{1-\frac{\mathrm{v}^{2}}{\mathrm{c}^{2}}}
$$

Dividing both sides of eq. 5 . by a small volume ' $v$ '.

$$
\frac{d f}{d v}=\frac{d q}{d v}\left[\gamma(v \times B)+E_{0}\right]
$$

Or

$$
\begin{aligned}
& d f=\left[\gamma(\rho v \times B)+E_{0}\right] d v \\
& d f=\left[\gamma(\mathcal{T} \times B)+E_{0}\right] d v
\end{aligned}
$$

Because $\rho v=\mathcal{T}$ ( current density) described in Maxwell's equation. To find total force acting on total charge enclosed by the surface $d s$ in the volume dv can be achieved by taking volume integral as.

$F=\int\left[\gamma(\mathcal{T} \times B)+E_{0}\right] d v$

\subsection{Electromagnetic force between two charges in moving inertial frame}

Consider two same charges are in rest frame S, only repulsive electrostatic force exists between them as shown in the fig. 1 .

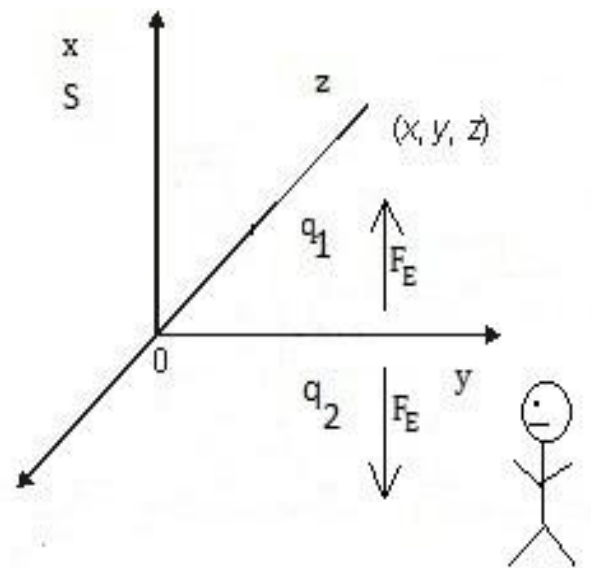

Fig. 1. Coulomb's force between two identical charges in rest frame $S$ measured by rest observer

If two same charges are in moving inertial frame $S$ ', each charge experiences repulsive electrostatic force, but due to the dumbbell shape magnetic field of two charges parallel to $\mathrm{x}-\mathrm{Z}$ plane in $S$ ' frame, they will attract each other with respect to rest observer as shown in fig. 2.

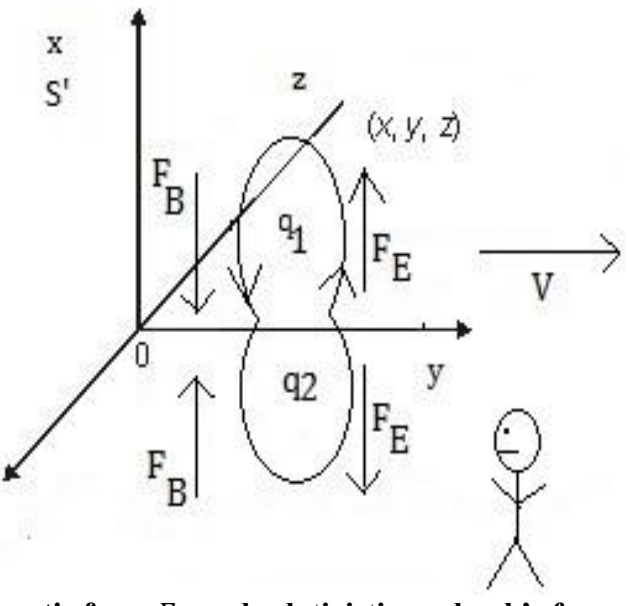

Fig. 2. Magnetic force $F_{B}$ and relativistic coulomb's force $F_{E}$ measured by rest observer when two identical charges are in moving inertial frame $S$ '

Here we are concerned with $S$ ' frame, to find the net force between two charges, we consider relativistic charge in coulomb's law as given in the following equation.

$\mathrm{F}_{\mathrm{E}}=\mathrm{qE}$

Magnetic field of a relativistic charge is given by.

$$
\mathrm{B}=\frac{\mu}{4 \pi \mathrm{r}^{2}} \mathrm{qv}
$$

Magnetic force on charge is given by.

$$
\mathrm{F}_{\mathrm{B}}=\mathrm{q}(\mathrm{V} \times \mathrm{B})
$$

Taking ratio between electric and magnetic force, we get the following result.

$$
\frac{F_{E}}{F_{B}}=\frac{v \times B}{E}=\frac{v^{2}}{c^{2}}
$$

Magnitude of charge in moving frame measured by rest observer is given by Asif's equation of charge variation.

$$
q=\frac{q_{o}}{\sqrt{1-\frac{v^{2}}{c^{2}}}}
$$


By re-arranging this equation, the value of $\frac{v^{2}}{c^{2}}$ can be determined as follows.

$\frac{\mathrm{v}^{2}}{\mathrm{c}^{2}}=1-\left(\frac{\mathrm{q}_{0}^{2}}{\mathrm{q}^{2}}\right)$

Substituting eq. 9. in eq. 8 . The result becomes

$$
\frac{\mathrm{v} \times \mathrm{B}}{\mathrm{E}}=1-\left(\frac{\mathrm{q}_{0}{ }^{2}}{\mathrm{q}^{2}}\right)
$$

After solving, we get the following equation.

$$
\mathrm{qE}=\mathrm{qvB}+\mathrm{q}_{0} \mathrm{E}_{0}
$$

This equation shows that coulomb's force (electric force) between two charges in $S^{\prime}$ frame measured by rest observer is the sum of the coulombs force between two charges in $S$ frame and magnetic force of two charges in S' frame.

And magnetic force between two charges in S' frame measured by rest observer is given by.

$$
\mathrm{qvB}=\mathrm{qE}-\mathrm{q}_{0} \mathrm{E}_{0}
$$

Eq. 11. Shows that the magnetic force between two charges in $S$ ' frame is actually the difference of the relativistic coulomb's force in S' frame and the rest coulomb's force in S frame.

From eq. 11. Whenv $=\mathrm{c}$, then

$$
\frac{\mathrm{qvb}}{\mathrm{qE}}=1
$$

When charges are in moving frame, the charge on the particle varies with respect to rest observer. By applying Faraday's law, one can deduct that the change in the electric charge causes change in the electric field and hence for rest observer, magnetic field is generated around each charge in moving frame. That's why the rest observer experience magnetic force between two charges during motion.

\section{MAXWELL'S EQUATIONS IN MOVING INERTIAL FRAME}

\subsection{Divergence of magnetic field of a point charge in moving frame}

In electromagnetic wave theory, the divergence of a magnetic field is always equal to zero because there is no mono pole magnet [6]. But in case of a single charge in moving inertial $\mathrm{S}$ ' frame along y-axis as shown in following Fig. 3 .

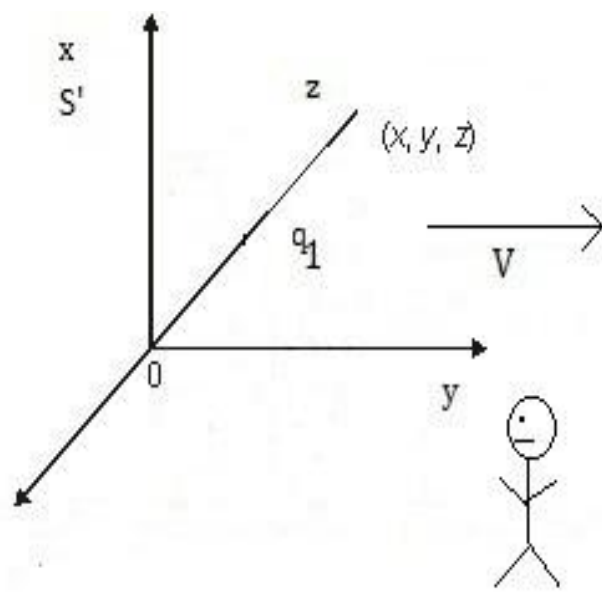

Fig. 3. Charge $q_{1}$ moving in moving inertial frame $S$, let us assume that it produce magnetic field in concentric circles around it with respect to rest observer. The divergence of that magnetic field measured by rest observer is given by.

$$
\nabla . \mathrm{B}=\lim _{\mathrm{v} \rightarrow 0} \frac{\oint \mathrm{B} \cdot \mathrm{ds}}{\mathrm{v}}
$$

Substituting $\quad B=\frac{\mu}{4 \pi r^{2}} q v$, For the continuous charge distribution in the small region of area $\mathrm{V} \rightarrow 0$, the above equation becomes

$$
\nabla . \mathrm{B}=\mu_{0} \rho \mathrm{v}
$$

$$
\nabla . \mathrm{B}=\mu_{0} \mathcal{T}
$$

Here is the relativistic current density, which depends upon both "Relativistic charge density and velocity". Note that Relativistic charge density varies with the change in the electric charge or change in the volume or both.

\subsubsection{Electric flux of Relativistic Electric field}

From Maxwell's point charge equation or Gausses law, one can deduct the total electric flux of charge out of the closed surface at rest which is $\frac{\rho}{\epsilon_{0}}$, suppose we want to demonstrate electric flux when charge is in moving inertial frame $S$ ' shown in above fig. 3. It follows from eq. 11. By eliminating relativistic charge and taking divergence to get total relativistic electric flux of charge.

$$
\begin{aligned}
& \nabla \cdot E=\lim _{V \rightarrow 0} \oiint_{S} \frac{E}{V} d s \\
& =\oiint_{S} \frac{(v \times B)}{V} d s+\gamma \oiint_{S} \frac{E_{0}}{V} d s
\end{aligned}
$$

Using divergence theorem to get electric flux. We have

$\oiint_{S} E d s=\mu_{0} \mathrm{qv}^{2}+\gamma \frac{\mathrm{q}_{0}}{\epsilon_{0}}$

$\oiint_{S} E d s=\frac{q^{2}}{\epsilon_{0} c^{2}}+\gamma \frac{q_{0}}{\epsilon_{0}}$

Putting value of gamma and after solving, we get

$\oiint_{S} E d s=\frac{\rho}{\epsilon_{0}}$

This is the point form of Maxwell's equation or Gausses law in integral form. Where ' $\rho$ ' is the relativistic charge density, which varies with the velocity of charges distributed in definite volume due to charge variation and length contraction according to special relativity.

\section{CONCLUSION}

In this study, Modified Electromagnetic theory has been proposed that enhance quantum electrodynamics and classical electromagnetic theory on the basis of charge variation. This leds to modify Lorentz force law, Maxwell's equations of point charge that gives correct result in rest frame will give different result in moving frames as discussed in this paper. The paper then discussed about the origin of electrostatic and electromagnetic forces between charges.

The interesting result found from coulomb's law is the factor $\mathrm{qc}^{2}$ which is the actual cause of attraction and repulsion between two charges in space as $\mathrm{mc}^{2}$ is the actual cause of gravitational force that appears in some Einstein's field 
equations that describe mass distorts space-time curvature in theory of General relativity.

\section{ACKNOWLEDGEMENTS}

The present study has been made on the basis of mathematical equations which describe Interpretation between Electromagnetic theory and Maxwell's equations.

\section{REFERENCES}

[1] J. C. Maxwell, "A Dynamical Theory of the Electromagnetic Field ", in W. D. Niven (ed.), The Scientific Papers of James Clerk Maxwell, New York, Dover, 1865.
[2] Laghari, Asif Ali. "Asif's Equation of Charge Variation and Special Relativity." Journal of Applied Physics, Vol. 4 No. 3, pp, 01-04, 2013.

[3] Wald, Robert M, "General relativity", University of Chicago press, 2010

[4] P. Lorrain and D.R. Corsou, "Electromagnetic Fields and Waves", San Francisco: Freeman, chs. 5 and 6, 1972.

[5] C.C. Su, "Modifications of the Lorentz force law invariant under Galilean transformations," in this Digest.

[6] R. E. Elliott,"Electromagnetics, History, Theory, and Applications", (classic reissue), 1993. 